\title{
On Two-channel Filter Banks with Directional Vanishing Moments
}

\author{
Arthur L. Cunha, Student Member, IEEE and Minh N. Do, Member, IEEE,
}

\begin{abstract}
The contourlet transform was proposed to address the limited directional resolution of the separable wavelet transform. In order to guarantee good nonlinear approximation behavior, the directional filters in the contourlet filter bank require sharp frequency response which incurs a large support size. We seek to isolate the key filter property that ensures good approximation. In this direction, we propose filters with directional vanishing moments (DVM). These filters, we show, annihilate information along a given direction. We study twochannel filter banks with DVM filters. We provide conditions under which the design of DVM filter banks is possible. A complete characterization of the product filter is thus obtained. We propose a design framework that avoids two-dimensional factorization using the mapping technique. The filters designed, when used in the contourlet transform, exhibit nonlinear approximation comparable to the conventional filters while being shorter and therefore providing better visual quality with less ringing artifacts.
\end{abstract}

Index Terms-Multidimensional Filter Banks, Biorthogonal Filter Banks, Nonseparable Filter Design, Directional Vanishing Moments, Contourlets, Nonlinear Approximation.

\section{INTRODUCTION}

$\mathbf{T}$ He separable discrete wavelet transform has established itself as a state-of-the art tool in several image processing applications, including compression, denoising, and feature extraction. A key property, that partially justifies the efficiency of wavelets in applications, is that it provides a sparse representation for several classes of images. Such sparsity can be precisely measured in some cases as the decay of the coefficients magnitude. For instance, for piecewise smooth images, the wavelet coefficients would decay as $O\left(N^{-1}\right)$. In spite of its high applicability, it is known that separable wavelets fail to explore the geometric regularity existent in most natural scenes, thus offering a suboptimal sparse representation. In this context, it is believed that the next generation transform coding compression algorithms will use a transform that better handles orientation and geometric information. In this direction, a number of researchers have proposed image representation schemes that achieve optimal sparsity behavior for some reasonable image model. Such is the case of the curvelet tight frames proposed by Candès and Donoho [1]. Inspired by curvelets, Do and Vetterli proposed the contourlet transform [2], which is a multiscale directional representation

A. L. Cunha is with the Department of Electrical and Computer Engineering and the Coordinated Science Laboratory, University of Illinois at UrbanaChampaign, Urbana IL 61801 (email: cunhada@uiuc.edu)

M. N. Do is with the Department of Electrical and Computer Engineering, the Coordinated Science Laboratory, and the Beckman Institute, University of Illinois at Urbana-Champaign, Urbana IL 61801 (email: minhdo@uiuc.edu).

This work was supported by a CAPES fellowship, Brazil, and the U.S. National Science Foundation under grant CCR-0237633 (CAREER). constructed in the discrete grid by combining the Laplacian pyramid [3], [4] and the directional filter bank (DFB) [5]. The distinctive feature in those constructions is that they are nonadaptive schemes. Furthermore, both are optimal for the piecewise smooth images in which the discontinuity is along a $C^{2}$ curve. In particular, under some conditions, the curvelet and contourlet transform coefficients magnitude would decay as $O\left(N^{-3 / 2}\right)$ for that class of images, which is the optimal rate [1], [2].

Geometric regularity in images exhibits through the fact that image edges are typically located along smooth contours. Thus, image singularities (i.e. edges) are localized in both location and direction. In order to extend 1-D wavelets crucial property for good approximation, namely vanishing moments [6], new 2-D representations like contourlets require a new condition named directional vanishing moment. For contourlets, such property can be imposed by carefully designing the refinement filters. Ideally, if the filters in the contourlet construction (see [2], [7] for details) are sinc-type filters with ideal response, then the contourlet atoms are guaranteed to have DVM's in an infinite number of directions. In practice however, ideal filters are approximated with a finite number of coefficients and to ensure DVM's, this number has to be large, thus increasing complexity. Alternatively, if FIR filters with enough DVM's can be obtained, one could achieve optimality of the approximation with shorter filters, which would result in a fast and efficient decomposition algorithm. In addition, as we learned from the wavelet experience, short filters (e.g., the filters chosen for the JPEG2000 standard) are very desirable for images as they are less affected by the Gibbs artefact.

In this paper we study two channel filter banks with DVM's. The DVM property leads to a new filter bank design problem and, to the best of our knowledge, this is the first work that addresses this problem. Two-channel filters banks are attractive since they are simpler to design and can be used in a tree structure to generate more complicated systems such as the DFB. Our goal is to impose directional vanishing moments in the contourlet basis function without resorting to long filters. That is, we attempt to cancel directional information using DVM's instead of good frequency selectivity, thus working with shorter filters and avoiding the Gibbs phenomena (see Figure 13). Potential applications of the filters designed in this work are in the contourlet transform of [2], the CRISPcontourlet system [8], and directionlets [9]. A preliminary version of the present work has appeared in [10].

The paper is structured as follows. In Section II we study filters with DVM and the class of signals that would be annihilated by such filter. In Section III we study the DVM in the context of 2-D FIR two-channel filter banks. We 
provide existence conditions as well as the design constraints. We also provide a complete characterization of the product filter of those filter banks. To overcome 2-D factorization, in Section IV we propose a design procedure using the mapping technique. The design is simple to carry out and uses the solution introduced in Section III. In Section V we study the use of filter banks with DVM in the contourlet construction. Experiments illustrating the approximation properties of the proposed filters are presented in Section VI and conclusions drawn in Section VII.

Notation: Throughout the paper we use boldface and capital boldface characters to represent two-dimensional (2-D) vectors and $2 \times 2$ matrices respectively. Thus, a discrete 2 -D signal is denoted by $x[\mathbf{n}]$ where $\mathbf{n}=\left(n_{1}, n_{2}\right)^{T}$. The 2-D $z$-transform of a signal $x[\mathbf{n}]$ is denoted by $X(\mathbf{z})$, where it is understood that $\mathbf{z}$ is shorthand for $\left(z_{1}, z_{2}\right)^{T}$. If $\mathbf{u}=\left(u_{1}, u_{2}\right)^{T}$ is a vector in $\mathbb{Z}^{2}$, then we denote $\mathbf{z}^{\mathbf{u}}=z_{1}^{u_{1}} z_{2}^{u_{2}}$ whereas, $\mathbf{z}^{\mathbf{S}}=\left(\mathbf{z}^{\mathbf{s}_{1}}, \mathbf{z}^{\mathbf{s}_{2}}\right)^{T}$ with the integer vectors $\mathbf{s}_{1}$ and $\mathbf{s}_{2}$ being the columns of the matrix $\mathbf{S}$. Note that with this notation, if $\mathbf{S}$ is a matrix of integers and $\mathbf{u}$ is an integer vector, then $\left(\mathbf{z}^{\mathbf{S}}\right)^{\mathbf{u}}=\mathbf{z}^{(\mathbf{S u})}$. A 1-D signal and its $z$-transform are denoted by $x[n]$ and $X(z)$ respectively.

\section{Directional Annihilating Filters}

Much of the efficiency of wavelets in analyzing transient signals is due to the vanishing moments in the wavelet function and its practical consequences [6]. Together with the time localization property, wavelets with vanishing moments provide a sparse representation for piecewise polynomial signals. Most successful wavelet filters such as the orthogonal Daubechies family and the JPEG2000 filters were designed with vanishing moment as a primary design criteria. This is in contrast to early filter bank constructions in which the frequency selectivity was a primary goal. Vanishing moments in a wavelet transform can be characterized by zeros in the highpass filters of the underlying filter bank. Suppose $H_{1}(z)$ is the highpass analysis filter of a two-channel filter bank. A vanishing moment of order $d$ is characterized by $d$ zeros at $z=1$ or $\omega=0$ on the unit circle. That is, the filter factors as $H_{1}(z)=(1-z)^{d} R_{1}(z)$. The filter $H_{1}(z)$ is related to discrete polynomial signals of degree less than $d-$ signals of the form $x[n]=\sum_{j=0}^{i} \alpha_{j} n^{j}$, with $\alpha_{j}$ real and $0 \leq i<d$. In particular, filtering $x[n]$ with $H_{1}(z)$ produces a zero output (see for example [11], [12]). In other words, the filter $H_{1}(z)$ totally annihilates discrete polynomials of degree less than $d$.

For 2-D filter banks with two channels, the vanishing moment concept can be generalized as point zeros at $\left(z_{1}, z_{2}\right)^{T}=$ $(1,1)^{T}$ or $\boldsymbol{\omega}=0$ [13], [14]. However, filters with point-zeros on the 2-D frequency plane do not cancel piecewise smooth images with discontinuities. A somewhat different philosophy motivated by contourlets is the directional vanishing moment in which the zeros are required to be along a line. Formally, we define DVM as follows.

Definition 1: Let $C(\mathbf{z})$ be a discrete filter and $\mathbf{u}=$ $\left(u_{1}, u_{2}\right)^{T}$ be a 2-D vector of coprime integers. We say $C(\mathbf{z})$ has a DVM of order $d$ along the direction $\mathbf{u}$ if it factors as

$$
C\left(z_{1}, z_{2}\right)=\left(1-z_{1}^{u_{1}} z_{2}^{u_{2}}\right)^{d} R\left(z_{1}, z_{2}\right) \text {. }
$$

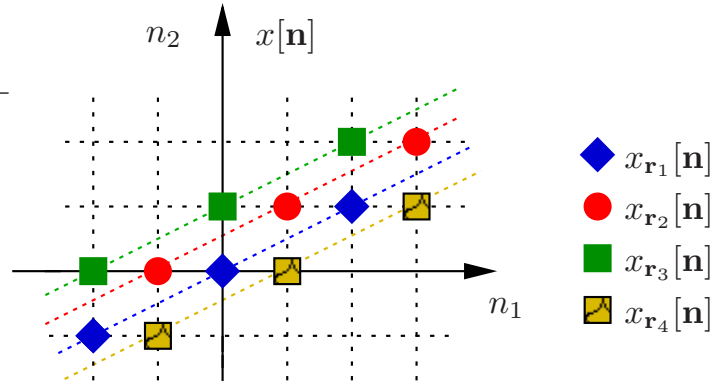

Fig. 1. The directional polyphase representation. Here $\mathbf{u}=(2,1)^{T}$ and $\mathbf{r}_{1}=$ $(0,0)^{T}, \mathbf{r}_{2}=(1,1)^{T}, \mathbf{r}_{3}=(0,1)^{T}$, and $\mathbf{r}_{4}=(1,0)^{T}$. The directional polyphase decomposition splits the signal into 1-D subsignals sampled along the direction $\mathbf{u}$. Those signals tile the whole 2-D discrete plane. We highlight in the picture some of the subsignals.

For contourlets, the filter $C(\mathbf{z})$ is a composite one, which involves the Laplacian pyramid filters and the polyphase components of the directional filters [2].

A question of interest is what signal would be annihilated (i.e., completely filtered out) by the filter in (1)? Such signal is an eigen-signal of the complementary branch of a two-channel filter bank where $C\left(z_{1}, z_{2}\right)$ is an analysis filter. This filter bank will be studied in detail in the next section. Similar to the 1-D case, 2-D filter banks with filters of the form in (1) have interesting properties with respect to approximation of smooth signals. In order to see those properties, we introduce the directional polyphase representation.

Lemma 1: Suppose that $\mathbf{u} \in \mathbb{Z}^{2}$ and $u_{2} \neq 0^{1}$. Then for every $\mathbf{n} \in \mathbb{Z}^{2}$ there exists a unique pair $(k, \mathbf{r})$ where $k \in \mathbb{Z}$, $\mathbf{r} \in \mathcal{R}:=\mathbb{Z} \times\left\{0,1, \ldots,\left|u_{2}\right|-1\right\}$ such that

$$
\mathbf{n}=k \mathbf{u}+\mathbf{r}
$$

Proof: Notice that (2) is equivalent to having $n_{1}=k u_{1}+$ $r_{1}$ and $n_{2}=k u_{2}+r_{2}$. For the second equation, $k$ and $r_{2}$ are uniquely determined as the quotient and remainder of $n_{2}$ divided by $u_{2}$. Given $k$, from the first equation, $r_{1}$ is also uniquely determined.

Lemma 1 allows us to partition any 2-D signal $x[\mathbf{n}]$ into a set of disjoint 1-D signals $\left\{x_{\mathbf{r}}[\mathbf{k}]\right\}_{\mathbf{k} \in \mathcal{R}}$ with

$$
x_{\mathbf{r}}[k]:=x[k \mathbf{u}+\mathbf{r}] .
$$

Figure 1 illustrates the directional polyphase representation. Note that each signal $x_{\mathbf{r}}[k]$ is a 1-D slice of $x[\mathbf{n}]$ along the direction $\mathbf{u}$. Therefore, the directional polyphase representation is distinct from the ordinary polyphase representation. Using Lemma 1, we can characterize the signals that are annihilated by the filter $C(\mathbf{z})$.

Proposition 1: Let $C\left(z_{1}, z_{2}\right)$ be a 2-D filter with a factor $\left(1-z_{1}^{u_{1}} z_{2}^{u_{2}}\right)^{d}$. Then a signal $x[\mathbf{n}]$ is annihilated by $C(\mathbf{z})$ if each 1-D signal $x_{\mathbf{r}}[k]$ defined in (3) is a discrete polynomial of degree less than $d$.

\footnotetext{
${ }^{1}$ Throughout the paper we assume $u_{2} \neq 0$. The case $u_{2}=0$ can be similarly handled by swapping the two variables $u_{1}, u_{2}$.
} 
Proof: Using Lemma 1 we have that

$$
\begin{aligned}
X(\mathbf{z}) & =\sum_{\mathbf{n} \in \mathbb{Z}^{2}} x[\mathbf{n}] \mathbf{z}^{-\mathbf{n}} \\
& =\sum_{k \in \mathbb{Z}} \sum_{\mathbf{r} \in \mathcal{R}} x[k \mathbf{u}+\mathbf{r}] \mathbf{z}^{-(k \mathbf{u}+\mathbf{r})} \\
& =\sum_{\mathbf{r} \in \mathcal{R}} \mathbf{z}^{-\mathbf{r}} \sum_{k \in \mathbb{Z}} x_{\mathbf{r}}[k] z^{-k \mathbf{u}} \\
& =\sum_{\mathbf{r} \in \mathcal{R}} \mathbf{z}^{-\mathbf{r}} X_{\mathbf{r}}\left(\mathbf{z}^{\mathbf{u}}\right) .
\end{aligned}
$$

Since the signals $x_{\mathbf{r}}[k]$ are polynomials of degree less than $d$, as in the 1-D case, each term $X_{\mathbf{r}}\left(\mathbf{z}^{\mathbf{u}}\right)$ is annihilated by a factor $\left(1-\mathbf{z}^{\mathbf{u}}\right)^{d}$. Thus it follows that $X(\mathbf{z})$ is annihilated by $C\left(z_{1}, z_{2}\right)$.

An immediate consequence of Proposition 1 is that discrete signals sampled from a continuous-time signal which is smooth away from line discontinuities along a given direction, are also annihilated by $C\left(z_{1}, z_{2}\right)$.

Corollary 1: Suppose that $x[\mathbf{n}]$ is the signal obtained by sampling a continuous time signal $x_{c}(\mathbf{t})$, that is,

$$
x[\mathbf{n}]=x_{c}(\Delta T \mathbf{n})
$$

where $x_{c}(\mathbf{t})$ is a piecewise polynomial of degree less than $d$ separated by lines along direction $\mathbf{u}$. Then $x[\mathbf{n}]$ is annihilated by a filter $C(\mathbf{z})$ with a factor $\left(1-\mathbf{z}^{\mathbf{u}}\right)^{d}$.

Proof: For each $\mathbf{r}$,

$$
x_{\mathbf{r}}[k]=x[k \mathbf{u}+\mathbf{r}]=x[\Delta T(k \mathbf{u}+\mathbf{r})]
$$

are the samples belonging to an entire polynomial region of $x_{c}(\mathbf{t})$, and thus $x_{\mathbf{r}}[k]$ is a polynomial signal of degree less than $d$.

As an illustration, we filter a piecewise smooth image (described by $e^{\frac{-x^{2}-y^{2}}{\alpha}}+\mathbf{1}_{\left\{\beta_{1}<y-2 x<\beta_{2}\right\}}$ ) with a 2-D filter with a third order DVM along direction $\mathbf{u}=(1,2)^{T}$. Such image is well approximated by a piecewise polynomial image of sufficiently large degree $d$. As can be seen in Figure 2, the edge was totally annihilated by the filtering operation.

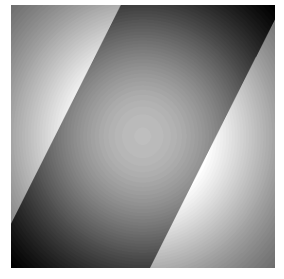

(a) Original

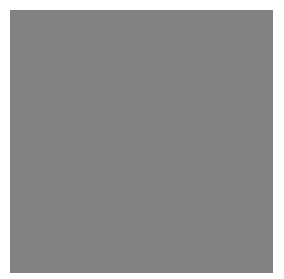

(b) Filtered
Fig. 2. Illustration of line zero moments as an edge annihilator. The piecewise polynomial image in (a) was filtered with a 2-D filter $C\left(z_{1}, z_{2}\right)=$ $\left(1-z_{1} z_{2}^{2}\right)^{3}$. The output image (b) pixels are approximately zero.

\section{TWO-CHANNEL FILTER BANKS WITH DIRECTIONAL VANISHING MOMENTS}

\section{A. Preliminaries}

Our setup consists in a general two-dimensional twochannel filter bank with a valid sampling $\mathbf{S}$ that has downsampling ratio 2, i.e., $\mid$ det $\mathbf{S} \mid=2$. Figure 4 (a) illustrates such filter bank. In this setting, given a set of analysis/synthesis filters $\left\{H_{0}(\mathbf{z}), H_{1}(\mathbf{z}), G_{0}(\mathbf{z}), G_{1}(\mathbf{z})\right\}$ the reconstructed signal is a perfect replica of the original provided that [15]

$$
\begin{aligned}
H_{0}(\mathbf{z}) G_{0}(\mathbf{z})+H_{1}(\mathbf{z}) G_{1}(\mathbf{z}) & =2,(4) \\
H_{0}\left(\mathbf{W}_{\mathbf{S}^{-T}}^{\mathbf{k}_{1}} \circ \mathbf{z}\right) G_{0}(\mathbf{z})+H_{1}\left(\mathbf{W}_{\mathbf{S}^{-T}}^{\mathbf{k}_{1}} \circ \mathbf{z}\right) G_{1}(\mathbf{z}) & =0
\end{aligned}
$$

where $\mathbf{k}_{1}$ is the nonzero integer vector in the set $\mathcal{N}(\mathbf{S}):=$ $\left\{\mathbf{S}^{T} x, x \in[0,1) \times[0,1)\right\}$ [15], and "o" denotes direct product between vector entries ${ }^{2}$. The modulation term $\mathbf{W}_{\mathbf{S}^{-T}}^{\mathbf{k}_{1}}$ [16] is a function of the sampling lattice generated by $\mathbf{S}$ and has the form $\mathbf{W}_{\mathbf{S}^{-T}}^{\mathbf{k}_{1}}=\left(e^{j \pi n_{1}}, e^{j \pi n_{2}}\right)^{T}$, where $\left(n_{1}, n_{2}\right)^{T}=$ $\mathbf{S}^{-T} \mathbf{k}_{1}$. Notice that all matrix generators of a given lattice are equivalent, up to right multiplication by a unimodular ${ }^{3}$ integer matrix [15]. The quincunx and the rectangular lattices are generated, for instance, by

$$
\mathbf{S}_{0}=\left(\begin{array}{cc}
1 & 1 \\
1 & -1
\end{array}\right) \text { and } \mathbf{S}_{1}=\left(\begin{array}{cc}
2 & 0 \\
0 & 1
\end{array}\right)
$$

respectively. The other possible lattice is the transpose of the rectangular and can be generated by exchanging the two diagonal entries of $\mathbf{S}_{1}$. It can be checked that $\mathbf{k}_{1}=(1,0)^{T}$ for both $\mathbf{S}_{0}$ and $\mathbf{S}_{1}$ so that $\mathbf{W}_{\mathbf{S}^{-T}}^{\mathbf{k}_{1}}=(-1,-1)^{T}$ and $\mathbf{W}_{\mathbf{S}^{-T}}^{\mathbf{k}_{1}}=$ $(-1,1)^{T}$ for $\mathbf{S}_{0}$ and $\mathbf{S}_{1}$ respectively. In what follows, we keep the rather heavy " $\mathbf{W}_{\mathbf{S}^{-T}}^{\mathbf{k}_{1}} \circ \mathbf{z}$ " notation whenever we wish to avoid having to treat the quincunx and rectangular cases separately.

Throughout the paper we assume FIR filters. In such case, using an argument similar to the one in [17], we can show the synthesis filters are completely determined (up to a scale factor and a delay) from the pair $\left(H_{0}(\mathbf{z}), G_{0}(\mathbf{z})\right)$ [17] through the relation

$\left(H_{1}(\mathbf{z}), G_{1}(\mathbf{z})\right)=\left(\mathbf{z}^{\mathbf{k}_{1}} G_{0}\left(\mathbf{W}_{\mathbf{S}^{-T}}^{\mathbf{k}_{1}} \circ \mathbf{z}\right), \mathbf{z}^{-\mathbf{k}_{1}} H_{0}\left(\mathbf{W}_{\mathbf{S}^{-T}}^{\mathbf{k}_{1}} \circ \mathbf{z}\right)\right)$.

As a result, the reconstruction condition reduces to

$$
H_{0}(\mathbf{z}) G_{0}(\mathbf{z})+H_{0}\left(\mathbf{W}_{\mathbf{S}^{-T}}^{\mathbf{k}_{1}} \circ \mathbf{z}\right) G_{0}\left(\mathbf{W}_{\mathbf{S}^{-T}}^{\mathbf{k}_{1}} \circ \mathbf{z}\right)=2 .
$$

The above biorthogonal relation specializes to the orthogonal one when $G_{0}(\mathbf{z})=H_{0}\left(\mathbf{z}^{-1}\right)$. Moreover, we say that $G_{0}(\mathbf{z})$ is the complementary filter to $H_{0}(\mathbf{z})$ whenever they satisfy (7).

\section{B. Two-channel filter banks with DVM's}

In general, given the desired direction of the zero moment, the analysis filter $H_{0}(\mathbf{z})$ takes the form

$$
H_{0}(\mathbf{z})=\left(1-z_{1}^{u_{1}} z_{2}^{u_{2}}\right)^{L} R_{H_{0}}(\mathbf{z}),
$$

where $L$ denote the order of the DVM. Substituting this in (7) we obtain the design equation

$$
\left(1-\mathbf{z}^{\mathbf{u}}\right)^{L} R(\mathbf{z})+\left(1-\left(\mathbf{W}_{\mathbf{S}^{-T}}^{\mathbf{k}_{1}} \circ \mathbf{z}\right)^{\mathbf{u}}\right)^{L} R\left(\mathbf{W}_{\mathbf{S}^{-T}}^{\mathbf{k}_{1}} \circ \mathbf{z}\right)=2,
$$

where $R(\mathbf{z}):=R_{H_{0}}(\mathbf{z}) G_{0}(\mathbf{z})$ is the complementary filter to $\left(1-\mathbf{z}^{\mathbf{u}}\right)^{L}$. We always assume that $u_{1}$ and $u_{2}$ are coprime integers. Note that the above relation sets a system

\footnotetext{
${ }^{2}$ That is, the $i$-th entry of $\mathbf{a} \circ \mathbf{b}$ is $a_{i} b_{i}$ where $a_{i}$ and $b_{i}$ are the $i$-th entries of $\mathbf{a}$ and $\mathbf{b}$ respectively

${ }^{3} \mathrm{~A}$ square matrix is unimodular if its determinant is equal to one.
} 
of linear equations which can be solved under certain conditions. In particular, it can be checked that $\left(\mathbf{W}_{\mathbf{S}^{-T}}^{\mathbf{k}_{1}} \circ \mathbf{z}\right)^{\mathbf{u}}=$ $e^{j 2 \pi \mathbf{u}^{T} \mathbf{S}^{-T} \mathbf{k}_{1}} \mathbf{z}^{\mathbf{u}}$. Since $\left|\operatorname{det} \mathbf{S}^{-T}\right|=|\operatorname{det} \mathbf{S}|^{-1}=1 / 2$, it follows that $\mathbf{u}^{T} 2 \mathbf{S}^{-T} \mathbf{k}_{1}$ is an integer scalar. If $\mathbf{u}^{T} 2 \mathbf{S}^{-T} \mathbf{k}_{1}$ is even, then a factor $\left(1-z^{\mathbf{u}}\right)$ exist in the two left terms of (8) in which case an FIR solution is not possible. Consequently, we see that $\mathbf{u}^{T} 2 \mathbf{S}^{-T} \mathbf{k}_{1}$ being odd is a necessary condition for solving (8). In that case, (8) reduces to

$$
\left(1-\mathbf{z}^{\mathbf{u}}\right)^{L} R(\mathbf{z})+\left(1+\mathbf{z}^{\mathbf{u}}\right)^{L} R\left(\mathbf{W}_{\mathbf{S}^{-T}}^{\mathbf{k}_{1}} \circ \mathbf{z}\right)=2 .
$$

Although in principle it is possible to solve (8) directly, the following Proposition simplifies the problem.

Proposition 2: Consider the filter equation (8) where $\mathbf{u}$ has coprime entries and $\mathbf{u}^{T} 2 \mathbf{S}^{-T} \mathbf{k}_{1}$ is odd. Then there exists a unimodular integer matrix $\mathbf{U}$ such that if $R(\mathbf{z})$ solves (8) then $\tilde{R}(\mathbf{z})=R\left(\mathbf{z}^{\mathrm{U}}\right)$ solves

$$
\left(1-z_{1}\right)^{L} \tilde{R}(\mathbf{z})+\left(1+z_{1}\right)^{L} \tilde{R}\left(\mathbf{W}_{\tilde{\mathbf{s}}^{-T}}^{\mathbf{k}_{1}} \circ \mathbf{z}\right)=2,
$$

where $\tilde{\mathbf{S}}=\mathbf{U S}$. Conversely, if $\tilde{R}(\mathbf{z})$ is a solution to (10) with $\tilde{\mathbf{S}}$ given and $\mathbf{u}$ as above, then there exists a matrix $\tilde{\mathbf{U}}$ such that $R(\mathbf{z})=\tilde{R}\left(\mathbf{z}^{\tilde{\mathbf{U}}}\right)$ is a solution to (8) with $\mathbf{S}=\tilde{\mathbf{U}} \tilde{\mathbf{S}}$.

Proof: We need to construct $\mathbf{U}$ so that $\left(\mathbf{z}^{\mathbf{U}}\right)^{\mathbf{u}}=\mathbf{z}^{\mathbf{U u}}=z_{1}$. We then set

$$
\mathbf{U}:=\left(\begin{array}{cc}
a & b \\
-u_{2} & u_{1}
\end{array}\right)
$$

and choose $a, b \in \mathbb{Z}$ so that $a u_{1}+b u_{2}=1$. Because $u_{1}$ and $u_{2}$ are assumed to be coprime, such $a$ and $b$ are guaranteed to exist. Since $\mathbf{u}^{T} 2 \mathbf{S}^{-T} \mathbf{k}_{1}$ is odd, substituting $\mathbf{z} \mapsto \mathbf{z}^{\mathbf{U}}$ in (9) and noting that

$$
\mathbf{W}_{\mathbf{S}^{-T}}^{\mathbf{k}_{1}} \circ\left(\mathbf{z}^{\mathbf{U}}\right)=\left(\mathbf{W}_{\mathbf{U}^{-T} \mathbf{S}^{-T}}^{\mathbf{k}_{1} \circ \mathbf{z}}\right)^{\mathbf{U}}
$$

gives (10). Conversely, if $\tilde{R}(\mathbf{z})$ solves (10), then set $\tilde{\mathbf{U}}=\mathbf{U}^{-1}$ with $\mathbf{U}$ as in (11) and substitute $\mathbf{z} \mapsto \mathbf{z}^{\tilde{\mathbf{U}}}$ in (10) to get (8).

Remark 1: 1. The fact that $\mathbf{U}$ has integer entries and is unimodular implies that it is a resampling matrix. Hence, the change of variables $\mathbf{z} \mapsto \mathrm{z}^{\mathrm{U}}$ or $\boldsymbol{\omega} \mapsto \mathbf{U}^{T} \boldsymbol{\omega}$ amounts to a resampling operation of the filter $R(\mathbf{z})$ which can be seen as a rearrangement of the filter coefficients in the 2-D discrete plane. This has the signal processing interpretation illustrated in Figure 3. Thus, we see that a filter with a DVM along a direction other than the horizontal one can be implemented in terms of a filter with a horizontal DVM plus pre/post resampling operations.

2. This change of variables can also be done in the filters of a filter bank. We thus obtain the equivalence shown in Figure 4 for a unimodular matrix $\mathbf{U}$ and $\tilde{\mathbf{S}}=\mathbf{U S}$. The equivalence can be easily checked using multirate identities. The filter bank within the dotted region in Figure 4 (b) is perfect reconstruction if and only if the filter bank in Figure 4 (a) is. Since the equivalence is one-to-one, one can design filter banks with horizontal DVM's and then, following Proposition 2, obtain filter banks with DVM's in any direction $\mathbf{u}$ such that $\mathbf{u}^{T} 2 \mathbf{S}^{-T} \mathbf{k}_{1}$ is odd.

3. Notice that a vertical DVM, i.e., a factor of the form $\left(1-z_{2}\right)^{L}$ could be obtained similarly, by just exchanging the rows of the matrix $\mathbf{U}$ constructed in the proof above.

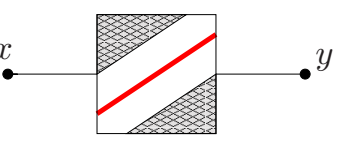

(a) $H(\mathbf{z})=\left(1-\mathbf{z}^{\mathbf{u}}\right)^{L} R(\mathbf{z})$

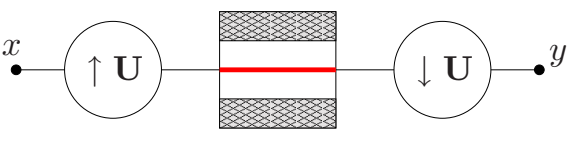

(b) $\tilde{H}(\mathbf{z})=H\left(\mathbf{z}^{\mathbf{U}}\right)=\left(1-z_{1}\right)^{L} \tilde{R}(\mathbf{z})$

Fig. 3. Change of variable is equivalent to a pre/pos resampling operation plus filtering with modified filter. (a) Filter with DVM along u. (b) Equivalent filtering structure with horizontal DVM.

Proposition 2 gives a simpler equation in the sense that a complete characterization of its solution is possible. Furthermore, with the aid of Proposition 2 we can establish a sufficient condition for solving (8) as the next proposition shows.

Proposition 3: Let $\mathbf{u}^{T}$ be an integer vector of co-prime integers. Then (8) admits an FIR solution if and only if $\mathbf{u}^{T} 2 \mathbf{S}^{-T} \mathbf{k}_{1}$ is an odd integer.

Proof: We already discussed necessity. To establish sufficiency, suppose that $\mathbf{u}^{T} 2 \mathbf{S}^{-T} \mathbf{k}_{1}$ is an odd integer. Using Proposition 2 , we can reduce the problem to that of (10). Thus if (10) is solvable then we are done. Now consider $\tilde{R}\left(\mathbf{W}_{\mathbf{U}^{-1} \mathbf{S}^{-T}}^{\mathbf{k}_{1}} \circ \mathbf{z}\right)$. If $\mathbf{W}_{\mathbf{U}^{-1} \mathbf{S}^{-T}}^{\mathbf{k}_{1}}$ has the form $(-1, \pm 1)$, then at least a univariate solution $R\left(z_{1}, z_{2}\right)=R\left(z_{1}\right)$ is guaranteed to exist [18]. But the first entry of $\mathbf{W}_{\mathbf{U}^{-1} \mathbf{S}^{-T}}^{\mathbf{k}_{1}}$ is given by $e^{j 2 \pi \mathbf{u}^{T} \mathbf{S}^{-T} \mathbf{k}_{1}}=-1$ since $2 \pi \mathbf{u}^{T} \mathbf{S}^{-T} \mathbf{k}_{1}$ is odd by assumption.

If $\mathbf{u}^{T} 2 \mathbf{S}^{-T} \mathbf{k}_{1}$ is an odd integer we then say that the direction $\mathbf{u}$ is admissible for the sampling matrix $\mathbf{S}$. Proposition 3 asserts that not all DVM's can be obtained for a given downsampling matrix $\mathbf{S}$. In particular, for the quincunx lattice generated by $\mathbf{S}_{0}$, we have that $\mathbf{u}^{T} 2 \mathbf{S}^{-T} \mathbf{k}_{1}=\left(u_{1}+u_{2}\right)$ so that $\mathbf{u}$ is admissible if $u_{1}+u_{2}$ is an odd integer. Similar for the rectangular lattice generated by $\mathbf{S}_{1}, \mathbf{u}^{T} 2 \mathbf{S}^{-T} \mathbf{k}_{1}=u_{1}$ so that $\mathbf{u}$ is admissible whenever $u_{1}$ is odd. For instance, $\mathbf{u}=(2,1)^{T}$ is admissible for $\mathbf{S}_{0}$ but not for $\mathbf{S}_{1}$, whereas $\mathbf{u}=(1,1)^{T}$ is only admissible for $\mathbf{S}_{1}$.

The aforementioned discussion provides necessary and sufficient conditions for having one of the branches of the filter bank featuring DVM's. In the context of contourlets (see Section V) it is desirable to have DVM's in both channels so that the DFB expansion tree is balanced in the sense that DVM's are present in all frequency channels. Unfortunately, this is not possible to attain with FIR filters. Likewise, it is neither possible to have different DVM's in the same filter channel. We summarize these assertions in the next proposition.

Proposition 4: Consider a two-channel 2-D filter bank with FIR filters $\left\{H_{0}(\mathbf{z}), H_{1}(\mathbf{z}), G_{0}(\mathbf{z}), G_{1}(\mathbf{z})\right\}$ and downsampling matrix $\mathbf{S}$. Let $\mathbf{u}=\left(u_{1}, u_{2}\right)^{T}$ and $\mathbf{v}=\left(v_{1}, v_{2}\right)^{T}$ be two distinct admissible directions. Then the filter bank cannot be perfect reconstruction if one of the following is true:

1. The filter $H_{0}(\mathbf{z})$ factors $\left(1-\mathbf{z}^{\mathbf{u}}\right)$ and $H_{1}(\mathbf{z})$ factors $(1-$ $\mathbf{z}^{\mathbf{v}}$ ) leaving FIR remainders.

2. One of the filters, say $H_{0}(\mathbf{z})$ factors $\left(1-\mathbf{z}^{\mathbf{u}}\right)$ and $\left(1-\mathbf{z}^{\mathbf{v}}\right)$ 


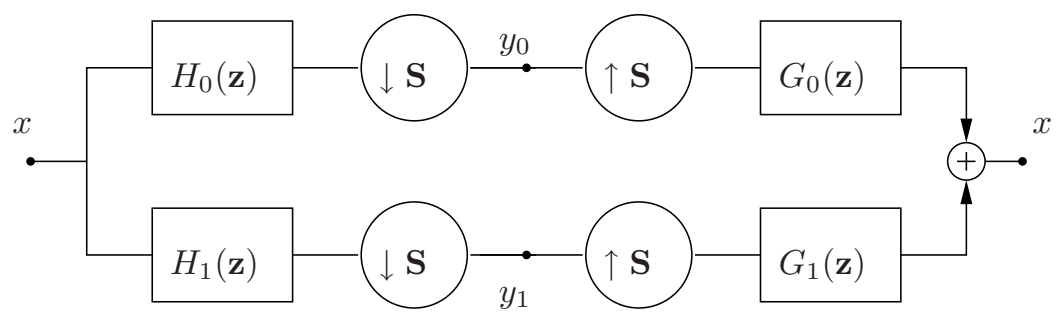

(a)

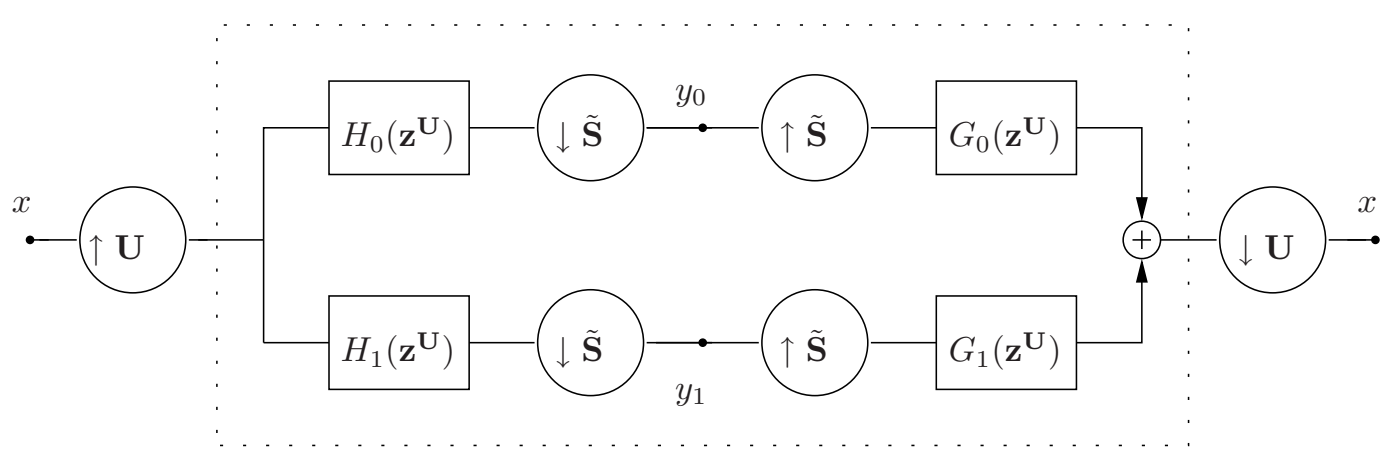

(b)

Fig. 4. Filter banks with DVM's along a fixed arbitrary direction are equivalent to a filter bank with DVM's along the horizontal direction. (a) Filter bank in which the filters have DVM's along the direction u. (b) The equivalent filter bank with DVM along horizontal direction. Note that U is constructed according to Proposition 2 and $\tilde{\mathbf{S}}=\mathbf{U S}$.

simultaneously leaving an FIR remainder.

Proof: 1. If the factor $\left(1-\mathbf{z}^{\mathbf{u}}\right)$ is in $H_{0}(\mathbf{z})$ and $\left(1-\mathbf{z}^{\mathbf{v}}\right)$ in $H_{1}(\mathbf{z})$, then the reconstruction condition (4) is violated on $\mathbf{z}=(1,1)^{T}$.

2. Suppose $H_{0}(\mathbf{z})$ factors $\left(1-\mathbf{z}^{\mathbf{u}}\right)$ and $\left(1-\mathbf{z}^{\mathbf{v}}\right)$. Because $\mathbf{v}$ is admissible, we have from Proposition 3 that $\mathbf{v}^{T} 2 \mathbf{S}^{-T} \mathbf{k}_{1}$ is odd. Consequently we have that

$$
\left(\mathbf{W}_{\mathbf{S}^{-T}}^{\mathbf{k}_{1}} \circ \mathbf{z}\right)^{\mathbf{v}}=-\mathbf{z}^{\mathbf{v}}
$$

It then follows from (6) that $G_{1}(\mathbf{z})$ factors $\left(1+\mathbf{z}^{\mathbf{v}}\right)$. Consider the system of equations $\left\{1-\mathbf{z}^{\mathbf{u}}=0,1+\mathbf{z}^{\mathbf{v}}=0\right\}$, which on the unit sphere becomes

$$
\left(\begin{array}{ll}
u_{1} & u_{2} \\
v_{1} & v_{2}
\end{array}\right)\left(\begin{array}{l}
\omega_{1} \\
\omega_{2}
\end{array}\right)=\left(\begin{array}{c}
\pi \\
2 \pi
\end{array}\right) .
$$

Because $\mathbf{u}$ and $\mathbf{v}$ are distinct we have that $u_{2} v_{1} \neq u_{1} v_{2}$ which guarantees a solution. It then follows that $H_{0}(\mathbf{z})$ and $G_{1}(\mathbf{z})$ have a common zero thus violating (4).

The previous proposition shows we can only afford to have DVM in one branch of the filter bank. In the next section, we present methods for solving the DVM filter bank design problem in the form of (10).

\section{Characterization of the Product Filter}

With the aid of Proposition 2 (see also Figure 4), in order to design filter banks with DVM's we need to consider (10) with two possible forms for $\tilde{R}\left(\mathbf{W}_{\mathbf{U}^{-1} \mathbf{S}^{-T}}^{\mathbf{k}_{1}} \circ \mathbf{z}\right)$, namely $\tilde{R}\left(-z_{1}, z_{2}\right)$ or $\tilde{R}\left(-z_{1},-z_{2}\right)$ corresponding to the rectangular and quincunx lattices. It turns out a complete characterization of the solution of (10) is possible as the next proposition shows.

Proposition 5: Let $s \in\{1,-1\}$. An FIR filter $\tilde{R}\left(z_{1}, z_{2}\right)$ is solution to the equation

$$
\left(1-z_{1}\right)^{L} \tilde{R}\left(z_{1}, z_{2}\right)+\left(1+z_{1}\right)^{L} \tilde{R}\left(-z_{1}, s z_{2}\right)=2
$$

if and only if it has the form

$$
\tilde{R}\left(z_{1}, z_{2}\right)=R_{L}\left(z_{1}\right)+\left(1+z_{1}\right)^{L} R_{o}\left(z_{1}, z_{2}\right)
$$

with $R_{L}\left(z_{1}\right)$ being a univariate solution given explicitly by

$$
R_{L}\left(z_{1}\right)=\sum_{i=0}^{L-1}\left(\begin{array}{c}
L+i-1 \\
L-1
\end{array}\right) 2^{-(L+i-1)}\left(1+z_{1}\right)^{i}
$$

and $R_{o}(\mathbf{z})$ satisfying

$$
R_{o}\left(z_{1}, z_{2}\right)+R_{o}\left(-z_{1}, s z_{2}\right)=0 .
$$

Proof: First, the 1-D complementary filter to $\left(1-z_{1}\right)^{L}$ is guaranteed to exist, a consequence of the Bezout theorem for polynomials [18]. Moreover, $R_{L}\left(z_{1}\right)$ as in (14) is the 1$\mathrm{D}$ minimum degree polynomial that solves (12), which can be found by Taylor series expansion [19]. Furthermore, if $R_{o}\left(z_{1}, z_{2}\right)$ satisfies $(15)$, it can be readily checked that $\tilde{R}(\mathbf{z})$ given in (13) solves (12).

To prove sufficiency, suppose $\tilde{R}\left(z_{1}, z_{2}\right)$ solves (12). Let $R^{\prime}\left(z_{1}, z_{2}\right):=\tilde{R}\left(z_{1}, z_{2}\right)-R_{L}\left(z_{1}\right)$. Since $R_{L}\left(z_{1}\right)$ and $\tilde{R}\left(z_{1}, z_{2}\right)$ are both solutions to (12) we must have

$$
R^{\prime}\left(-z_{1}, s z_{2}\right)\left(1+z_{1}\right)^{L}=-R^{\prime}\left(z_{1}, z_{2}\right)\left(1-z_{1}\right)^{L},
$$


which implies that $R^{\prime}\left(z_{1}, z_{2}\right)=\left(1+z_{1}\right)^{L} R_{o}\left(z_{1}, z_{2}\right)$. Now, let $z_{1} \neq \pm 1$. Then (16) implies (15) and since $R_{o}(\mathbf{z})$ is an FIR filter, it follows that (15) is valid for all $\mathbf{z} \in \mathbb{C}^{2}$.

Remark 2: 1. The above proposition is akin to its 1-D counterpart which is used to construct compactly supported wavelets (see e.g., [19]). The distinction occurs in the higher order term $R_{o}\left(z_{1}, z_{2}\right)$ which now can be any two-dimensional function satisfying (15). This higher order term will make the filter a "truly" 2-D one, meaning a filter with a nonseparable support. Moreover, the higher order term can be used to control the shape of the 2-D frequency response.

2. If $L$ is even, then it is easy to check that

$$
\begin{aligned}
\tilde{R}\left(z_{1}, z_{2}\right) & =\left(-2 z_{1}\right)^{-L / 2} R_{L / 2}\left(\frac{z_{1}+z_{1}^{-1}}{2}\right) \\
& +\left(1+z_{1}^{-1}\right)^{L} R_{o}\left(z_{1}, z_{2}\right)
\end{aligned}
$$

with $R_{o}\left(z_{1}, z_{2}\right)$ satisfying (15) also solves (12). If in addition $R_{o}\left(z_{1}, z_{2}\right)=R_{o}\left(z_{1}^{-1}, z_{2}^{-1}\right)$, this solution provides a class of linear-phase biorthogonal filters with DVM in which each one of the filters in the analysis and synthesis is a degenerate 1-D solution. Thus, this solution can be seen as a 2-D generalization of the 1-D biorthogonal spline wavelet filters of [19].

The result also extends to the orthogonal case as the next corollary shows.

Corollary 2: Let $s$ as in Proposition 5. Consider the orthogonal perfect reconstruction condition

$H_{0}\left(z_{1}, z_{2}\right) H_{0}\left(z_{1}^{-1}, z_{2}^{-1}\right)+H_{0}\left(-z_{1}, s z_{2}\right) H_{0}\left(-z_{1}^{-1}, s z_{2}^{-1}\right)=2$, with $H_{0}\left(z_{1}, z_{2}\right)=\left(1-z_{1}^{-1}\right)^{L} r_{0}\left(z_{1}, z_{2}\right)$. Also set $R_{0}\left(z_{1}, z_{2}\right)=$ $r_{0}\left(z_{1}, z_{2}\right) r_{0}\left(z_{1}^{-1}, z_{2}^{-1}\right)$. Then $R_{0}\left(z_{1}, z_{2}\right)$ has the form

$R_{0}\left(z_{1}, z_{2}\right)=R_{L}\left(\frac{z_{1}+z_{1}^{-1}}{2}\right)+\left(1+\frac{z_{1}+z_{1}^{-1}}{2}\right)^{L} \tilde{R}_{0}\left(z_{1}, z_{1}\right)$,

where $R_{L}$ is as in (14), $\tilde{R}_{0}\left(z_{1}, z_{2}\right)$ satisfies (15), and $\tilde{R}_{0}\left(z_{1}, z_{2}\right)=\tilde{R}_{0}\left(z_{1}^{-1}, z_{2}^{-1}\right)$.

The proof of this corollary is a direct application of Proposition 5. Notice that orthogonal FB's with DVM's could be obtained using the above result by taking the square root of the filter $R_{0}\left(e^{j \omega}\right)=\left|r_{0}\left(e^{j \omega}\right)\right|^{2}$. This requires 2-D spectral factorization, which is a hard task. Furthermore, such a square root is not guaranteed to exist and one has to carefully select the higher order term $\tilde{R}_{0}\left(e^{j \omega}\right)$ so as to make $R_{0}\left(e^{j \omega}\right)$ factorizable. For biorthogonal solutions, one can avoid spectral factorization using the mapping approach as discussed in the next section.

\section{DESIGN VIA MAPPING}

\section{A. Design Procedure}

Due to the lack of a factorization theorem for 2-D polynomials, the design of nonseparable 2-D filter banks is substantially harder than the 1-D counterpart. In particular, we cannot easily factor the solution for the product filter given by Proposition 5 into $H_{0}(\mathbf{z})$ and $G_{0}(\mathbf{z})$ as in (7). There are two known ways to avoid factorization: (1) Constructing the polyphase matrix in a lattice structure and (2) Mapping 1-D filters to 2-D by appropriate change of variables. Most filters designed in the literature use one of these two approaches (see e.g., [20], [21], [22], [23], [24].) The first method has the attractive feature of possible construction of both orthogonal and biorthogonal solutions. However, it is harder to impose vanishing moments, since the corresponding conditions in the polyphase domain are nonlinear (see for e.g., [25].) For processing images, orthogonal FB's have the shortcoming of lack of phase linearity which causes severe visual distortions. For biorthogonal FIR solutions, one can use the general mapping approach proposed in [23]. In this approach, we first design 1-D prototype filters $H_{0}^{(1 \mathrm{D})}(z)$, and $G_{0}^{(1 \mathrm{D})}(z)$, such that $P^{(1 \mathrm{D})}(z):=H_{0}^{(1 \mathrm{D})}(z) G_{0}^{(1 \mathrm{D})}(z)$ is a halfband filter, i.e.,

$$
P^{(1 \mathrm{D})}(z)+P^{(1 \mathrm{D})}(-z)=2 .
$$

Next, we apply the change of variables $z \mapsto M(\mathbf{z})$ to map the 1-D filters to 2-D ones:

$$
H_{0}(\mathbf{z})=H_{0}^{(1 \mathrm{D})}(M(\mathbf{z})), G_{0}(\mathbf{z})=G_{0}^{(1 \mathrm{D})}(M(\mathbf{z})) .
$$

It can be easily checked that the mapped 2-D filters will satisfy the perfect reconstruction condition (7) provided

$$
M(\mathbf{z})=-M\left(\mathbf{W}_{\mathbf{S}^{-T}}^{\mathbf{k}_{1}} \circ \mathbf{z}\right) .
$$

Notice that for FIR solutions, it is necessary that the 1D prototype filters have only positive powers of $z$. This automatically precludes FIR orthogonal solutions.

Mapping 1-D filters can also be carried over to the polyphase domain as done in [24]. A more careful examination of the filters proposed in [24] reveals that the polyphase mapping can also be performed in the filter domain, and as such, the technique boils down to a particular case of [23]. For completeness, we include a detailed discussion of this in the appendix.

In the context of filter banks with DVM's, the goal is to devise a mapping function $M(\mathbf{z})$ such that each of the 2-D filters $H_{0}(\mathbf{z})$, and $G_{0}(\mathbf{z})$ has a given number of $\left(1-z_{1}\right)$ factors. In addition we require $M(\mathbf{z})$ so that perfect reconstruction is kept after mapping. The next proposition shows an explicit form of the required mapping function.

Proposition 6: Let $H_{0}^{(1 \mathrm{D})}(z), G_{0}^{(1 \mathrm{D})}(z)$ be such that $P^{(1 \mathrm{D})}(z)=H_{0}^{(1 \mathrm{D})}(z) G_{0}^{(1 \mathrm{D})}(z)$ satisfies (18) and let $s \in\{1,-1\}$. Suppose $M(\mathbf{z})$ is an FIR mapping function such that

$$
M\left(z_{1}, z_{2}\right)=\left(1-z_{1}\right)^{L} \tilde{R}\left(z_{1}, z_{2}\right)+c_{0},
$$

where $c_{0}$ is such that $H_{0}^{(1 \mathrm{D})}(z)$ has a factor $\left(z-c_{0}\right)^{N_{a} / L}$, $G_{0}^{(1 \mathrm{D})}(z)$ has a factor $\left(z-c_{0}\right)^{N_{s} / L}$, and $\tilde{R}\left(z_{1}, z_{2}\right)$ satisfies the valid mapping equation:

$$
\left(1-z_{1}\right)^{L} \tilde{R}\left(z_{1}, z_{2}\right)+\left(1+z_{1}\right)^{L} \tilde{R}\left(-z_{1}, a z_{2}\right)=2 c_{0} .
$$
Then

1. The mapped filters $H_{0}(\mathbf{z})=H_{0}^{(1 \mathrm{D})}(M(\mathbf{z}))$ and $G_{0}(\mathbf{z})=$ $G_{0}^{(1 \mathrm{D})}(M(\mathbf{z}))$ are perfect reconstruction, i.e., they satisfy

$$
H_{0}\left(z_{1}, z_{2}\right) G_{0}\left(z_{1}, z_{2}\right)+H_{0}\left(-z_{1}, s z_{2}\right) G_{0}\left(-z_{1}, s z_{2}\right)=2 .
$$

2. The mapped filters factor as

$$
H_{0}(\mathbf{z})=\left(1-z_{1}\right)^{N_{a}} R_{H_{0}}(\mathbf{z}), \quad G_{0}(\mathbf{z})=\left(1-z_{1}\right)^{N_{s}} R_{G_{0}}(\mathbf{z}) .
$$


Proof: Suppose $M(\mathbf{z})$ is as in (20). Then, from (21) it follows that $M\left(z_{1}, z_{2}\right)=-M\left(-z_{1}, s z_{2}\right)$ and from (19) the mapped filters satisfy (22). Moreover, substituting $z \mapsto M(\mathbf{z})$ in the factors $\left(z-c_{0}\right)^{N_{a} / L}$ of $H_{0}^{(1 \mathrm{D})}(z)$ and $\left(z-c_{0}\right)^{N_{s} / L}$ of $G_{0}^{(1 \mathrm{D})}(z)$ gives $H_{0}(\mathbf{z})$ and $G_{0}(\mathbf{z})$ as in (23).

Interestingly, it turns out the valid mapping equation (21) is similar to equation (12) for the product filter in Proposition 5. We then can use Proposition 5 to find an explicit solution to (21). In short, we see that the mapping overcomes the need for spectral factorization and, together with Proposition 5, gives a straightforward design methodology. Hence, we can formulate the design of DVM filter banks via mapping as follows.

Problem: design 2-D filters $H_{0}^{(1 \mathrm{D})}(\mathbf{z})$ and $G_{0}^{(1 \mathrm{D})}(\mathbf{z})$ satisfying the perfect reconstruction condition (7) and such that $H_{0}^{(1 \mathrm{D})}(\mathbf{z})$ factors $\left(1-z_{1}\right)^{N_{a}}$ and $G_{0}^{(1 \mathrm{D})}(\mathbf{z})$ factors $\left(1-z_{1}\right)^{N_{s}}$.

Step 1 Design 1-D filters $H_{0}^{(1 \mathrm{D})}(z)$ and $G_{0}^{(1 \mathrm{D})}(z)$ with $N_{a} / L$ and $N_{s} / L$ zeros at some point $c_{0} \in \mathbb{C}$ respectively, and such that $P^{(1 \mathrm{D})}(z)=H_{0}^{(1 \mathrm{D})}(z) G_{0}^{(1 \mathrm{D})}(z)$ satisfies (18).

Step 2 Let $M(\mathbf{z})=\left(1-z_{1}\right)^{L} \tilde{R}(\mathbf{z})+c_{0}$ with

$$
\begin{gathered}
\tilde{R}(\mathbf{z})=\tilde{R}_{L}\left(z_{1}\right)+\left(1+z_{1}\right)^{L} \tilde{R}_{o}\left(z_{1}, z_{2}\right), \text { and } \\
\tilde{R}_{o}\left(z_{1}, z_{2}\right)=-\tilde{R}_{o}\left(-z_{1}, s z_{2}\right) .
\end{gathered}
$$

Step 3 Set $H_{0}(\mathbf{z})=H_{0}^{(1 \mathrm{D})}(M(\mathbf{z}))$ and $G_{0}(\mathbf{z})=$ $G_{0}^{(1 \mathrm{D})}(M(\mathbf{z}))$ to obtain the desired 2-D filters.

Notice that one can choose $M(\mathbf{z})$ so that $M(\mathbf{z})=M\left(\mathbf{z}^{-1}\right)$ and, as a result, the 2-D filters are zero-phase [23]. In this case, $L$ is necessarily even and $\tilde{R}(\mathbf{z})$ can have the more convenient form in (17) instead of the one in (13). In the design examples that follow we use $c_{0}=1$. It is easy to check that this ensures the gain of $H_{1}(\mathbf{z})$ at $\mathbf{z}=\left(1, z_{2}\right)^{T}$ is $\sqrt{2}$ whenever $H_{0}^{(1 \mathrm{D})}(-1)=\sqrt{2}$.

\section{B. Filter Size Analysis}

One shortcoming of the mapping design procedure is that the size of the support of the filters tends to be increasingly large. However, if extra care is taken when designing the mapping function as well as the 1-D prototypes, the filters can have reasonable support size. The support of the filters can be easily quantified as we show next. We use the notation $\operatorname{deg}[\cdot]$ to denote the support size of the filter. For a 2-D filter the support size will be a pair of integers that represent the sides of the smallest discrete square that contains all the filter coefficients, including the boundary. Thus, since $H_{0}(\mathbf{z})=H_{0}^{(1 \mathrm{D})}(M(\mathbf{z})), G_{0}(\mathbf{z})=G_{0}^{(1 \mathrm{D})}(M(\mathbf{z}))$, we have that $\operatorname{deg}\left[H_{0}(\mathbf{z})\right]=\operatorname{deg}\left[H^{(1 \mathrm{D})}(z)\right] \operatorname{deg}[M(\mathbf{z})]$ and similarly $\operatorname{deg}\left[G_{0}(\mathbf{z})\right]=\operatorname{deg}\left[G^{(1 \mathrm{D})}(z)\right] \operatorname{deg}[M(\mathbf{z})]$. Notice that $\operatorname{deg}[M(\mathbf{z})]=\operatorname{deg}[R(\mathbf{z})]+(L, 0)^{T}$. Moreover, from Proposition 5 we have that $\tilde{R}\left(z_{1}, z_{2}\right)=R_{L}\left(z_{1}\right)+\left(1+z_{1}\right)^{L} R_{o}(\mathbf{z})$. Since $R_{L}\left(z_{1}\right)$ is the minimum degree complementary filter to $\left(1-z_{1}\right)^{L}$, it has support size $L$. Therefore, if we further assume that $R_{o}(\mathbf{z})$ is supported around the origin, it follows that $\operatorname{deg}\left[\tilde{R}\left(z_{1}, z_{2}\right)\right]$ is dominated by $\operatorname{deg}\left[\left(1+z_{1}\right)^{L} R_{o}(\mathbf{z})\right]$. Thus, denoting $\operatorname{deg}\left[R_{o}(\mathbf{z})\right]=\left(\mu_{1}, \mu_{2}\right)^{T}$, we have that

$$
\operatorname{deg}\left[\tilde{R}\left(z_{1}, z_{2}\right)\right] \leq \operatorname{deg}\left[\left(1+z_{1}\right)^{L} R_{o}(\mathbf{z})\right]=\left(\begin{array}{c}
L+\mu_{1} \\
\mu_{2}
\end{array}\right),
$$

and consequently,

$$
\operatorname{deg}\left[H_{0}\left(z_{1}, z_{2}\right)\right] \leq \operatorname{deg}\left[H^{(1 \mathrm{D})}(z)\right]\left(\begin{array}{c}
\mu_{1}+2 L \\
\mu_{2}
\end{array}\right) .
$$

Similarly, for the synthesis filter:

$$
\operatorname{deg}\left[G_{0}\left(z_{1}, z_{2}\right)\right] \leq \operatorname{deg}\left[G^{(1 \mathrm{D})}(z)\right]\left(\begin{array}{c}
\mu_{1}+2 L \\
\mu_{2}
\end{array}\right) .
$$

From the foregoing discussion we see that when $\mu_{1} \simeq \mu_{2}$, by increasing the number of DVM's in the mapping function, i.e., increasing $L$, the filter support will be stretched along the $n_{1}$ direction. Furthermore, for a fixed mapping function, the support of the resulting 2-D filter will increase linearly in both $n_{1}, n_{2}$ directions with the number of vanishing moments in the prototype filters $H^{(1 \mathrm{D})}(z)$. Thus, to avoid the filters being too large, we point out that the 1-D prototype filters should be as short as possible, preferably with only one zero at $c_{0}$. We present design examples next.

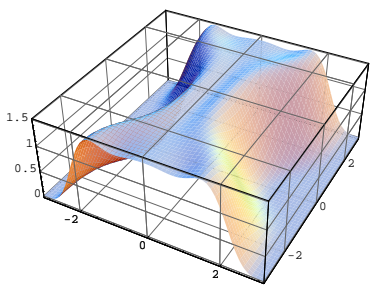

(a) $\left|H_{0}\left(e^{j \boldsymbol{\omega}}\right)\right|$

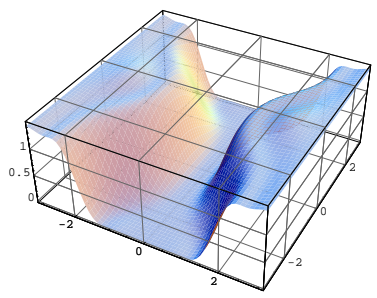

(c) $\mid H_{1}\left(e^{j \boldsymbol{\omega}}\right)$

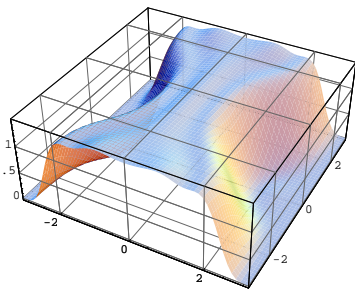

(b) $\left|G_{0}\left(e^{j \boldsymbol{\omega}}\right)\right|$

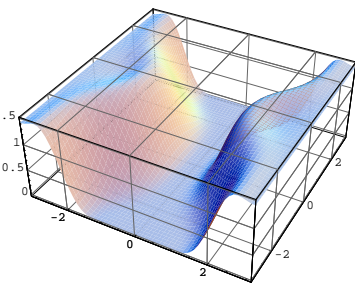

(d) $\left|G_{1}\left(e^{j \boldsymbol{\omega}}\right)\right|$
Fig. 5. Frequency response of analysis and synthesis filters designed with 4-th order directional vanishing moment. The filters degenerate to the 9-7 filters.

\section{Design Examples}

Example 1: Nonseparable filter family that includes the 1D 9-7 filters

For the purpose of this example we assume the quincunx lattice and we generate DVM's along the horizontal direction $u_{1}=0$. Following the discussion in the previous section we choose the prototype filters $H_{0}^{(1 \mathrm{D})}(z)$ and $G_{0}^{(1 \mathrm{D})}(z)$ to have zeroes at $z=-1$. We consider the minimum degree complementary filter to $(1-z)^{4}$ which from (14) gives the product filter

$$
P^{(1 \mathrm{D})}(z)=\frac{1}{16}\left(16-29 z+20 z^{2}-5 z^{3}\right)(1-z)^{4} .
$$


We let each prototype filter have a factor $(1-z)^{2}$ and then we split the factor $\left(16-29 z+20 z^{2}-5 z^{3}\right)$ between the two prototypes assigning the real root to $H_{0}^{(1 \mathrm{D})}(z)$ and the two complex-conjugate roots to $G_{0}^{(1 \mathrm{D})}(z)$.

In the mapping function we impose the condition $M\left(z_{1}, z_{2}\right)=M\left(z_{1}^{-1}, z_{2}^{-1}\right)$ so that the filters are zero-phase. Following (20), in order to generate a second order horizontal DVM we set

$$
M\left(z_{1}, z_{2}\right)=\left(1-z_{1}\right)^{2} R\left(z_{1}, z_{2}\right)-1 .
$$

To guarantee that the map satisfies the valid mapping condition (21) and is zero-phase we use (17) to obtain

$$
R\left(z_{1}, z_{2}\right)=-\frac{z_{1}^{-1}}{2}+\left(1+z_{1}^{-1}\right)^{2} R_{o}\left(z_{1}, z_{2}\right) .
$$

Notice that $R_{o}\left(z_{1}, z_{2}\right)$ can be any zero-phase filter that satisfies (15). For simplicity we choose $R_{o}\left(z_{1}, z_{2}\right)=\alpha\left(z_{2}+\right.$ $\left.z_{2}^{-1}\right)$. With $\alpha=0$ we recover the 9-7 filters. Figure 5 displays the frequency response of the filters when we set $\alpha=-4 \sqrt{2}$.

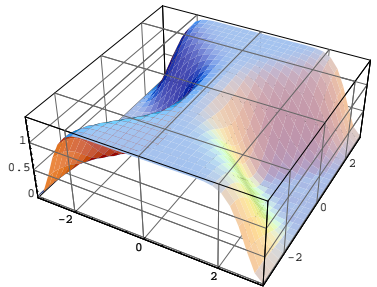

(a) $\left|H_{0}\left(e^{j \boldsymbol{\omega}}\right)\right|$

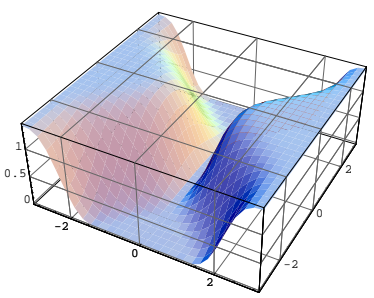

(c) $\left|H_{1}\left(e^{j \boldsymbol{\omega}}\right)\right|$

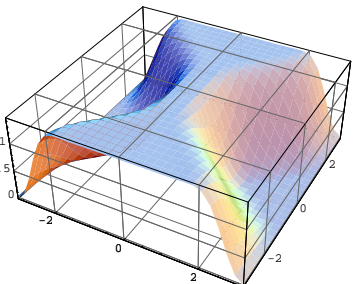

(b) $\left|G_{0}\left(e^{j \omega}\right)\right|$

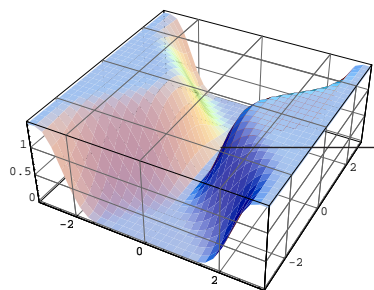

(d) $\left|G_{1}\left(e^{j \boldsymbol{\omega}}\right)\right|$
Fig. 6. Frequency response of analysis and synthesis filters designed with 2-th order directional vanishing moment.

Example 2: Using the higher order term to improve frequency response

In this example we use the extra degrees of freedom in our proposed design to obtain filters with low order DVM's and better frequency selectivity. The following filters can be checked to satisfy (18):

$$
\begin{aligned}
& H_{0}^{(1 \mathrm{D})}(z)=K\left(1+k_{2} z+k_{2} k_{3} z^{2}\right), \\
& G_{0}^{(1 \mathrm{D})}(z)=K^{-1}\left(1-k_{1} z-k_{3} z+k_{1} k_{2} z^{2}-k_{1} k_{2} k_{3} z^{3}\right) .
\end{aligned}
$$

To obtain the prototype we choose the constants $K, k_{1}, k_{2}$, and $k_{3}$ such that each filter has a zero at $z=-1$ and in addition that $H_{0}^{(1 \mathrm{D})}(-1)=G_{0}^{(1 \mathrm{D})}(-1)=\sqrt{2}$. The following prototypes are obtained:

$$
\begin{aligned}
H_{0}^{(1 \mathrm{D})}(z) & =\frac{1}{2}(1-z)(2+(2-\sqrt{2}) z) \\
G_{0}^{(1 \mathrm{D})}(z) & =\frac{1}{2}(1-z)\left(2+(6-4 \sqrt{2}) z+(4-3 \sqrt{2}) z^{2}\right) .
\end{aligned}
$$

We then use the same mapping function of Example 1, but now we let

$$
\begin{aligned}
R_{o}\left(z_{1}, z_{2}\right) & =r_{0}\left(z_{1}+\frac{1}{z_{1}}\right)+r_{1}\left(z_{2}+\frac{1}{z_{2}}\right) \\
& +r_{2}\left(z_{1}+\frac{1}{z_{1}}\right)\left(z_{2}+\frac{1}{z_{2}}\right)^{2}
\end{aligned}
$$

and optimize the coefficients $r_{0}, r_{1}, r_{2}$ so that the filters approximate the ideal fan response. The resulting filters $H_{0}(\mathbf{z})$ and $G_{0}(\mathbf{z})$ have sizes $13 \times 9$ and $19 \times 13$ respectively. Figure 6 displays the frequency response of all the filters.

\section{Tree-Structured Filter Banks With DiReCTIONAL VANISHING MOMENTS}

In order to study the approximation properties of DVM filters we replace the conventional DFB in the contourlet transform with a DFB constructed with fan filter banks that have DVM's. The DFB is constructed with fan filter banks and pre/post resampling operations in a tree structure [5]. Referring to the fan-shaped fundamental frequency support, it is natural to impose vanishing moments along the vertical and horizontal directions. In fact, as demonstrated in [26], a high order DVM along the direction orthogonal to the contourlet element main orientation is sufficient for ensuring optimal approximation property.

As we already discussed in Proposition 4, for a 2-D, twochannel filter bank, we can only have DVM's in one of its branches. Thus, for the prototype fan filter bank used in the DFB, we have the two possible configurations (denoted type 0 and type 1) with similar frequency decomposition illustrated in Figure 7.

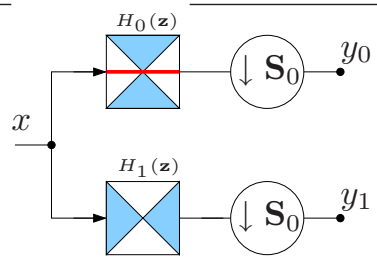

(a) Type 0

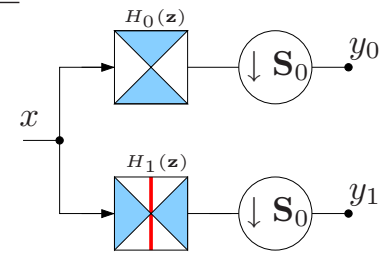

(b) Type 1
Fig. 7. Two types of prototype fan filter banks used in the DFB expansion tree. Each filter bank has one of its branches featuring a DVM.

In light of that, in each node of the DFB tree structure we use a sheared/rotated filter bank, according to the DFB expansion rule, obtained from the prototype fan filter bank of either type 0 or type 1 . This naturally opens the question on how to arrange the filter bank types in the tree structure in an efficient way. Notice that, for a DFB with $l$ stages, a total of $\prod_{i=1}^{l} 2^{i}$ tree arrangements are possible, each with a different DVM allocation among the DFB channels. Figure 8 illustrates a possible arrangement for a four-channel DFB. Notice that with type 0 and type 1 prototype fan filter banks we obtain DVM's in different directions (in this case the two diagonal directions)

For a single node in the DFB tree, the possible DVM's for either type 0 or type 1 fan filter bank to be appended in that node will depend on the overall downsampling matrix of that 


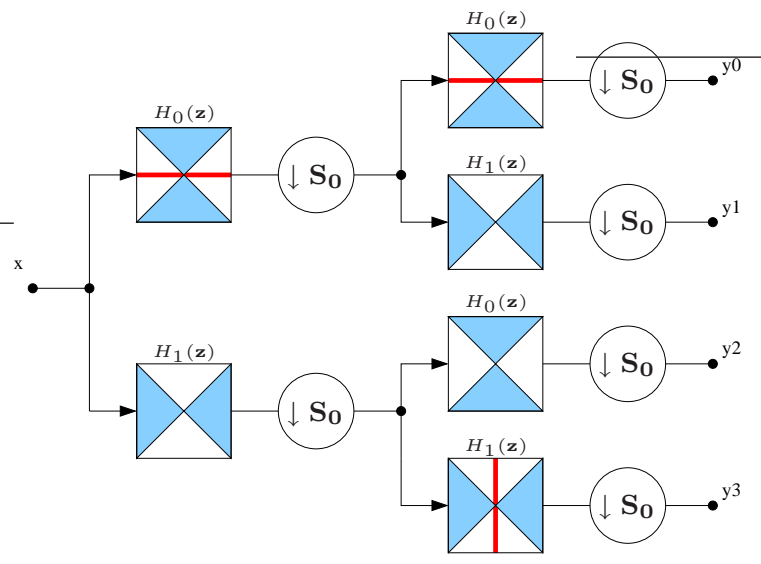

(a) DFB with DVM filters

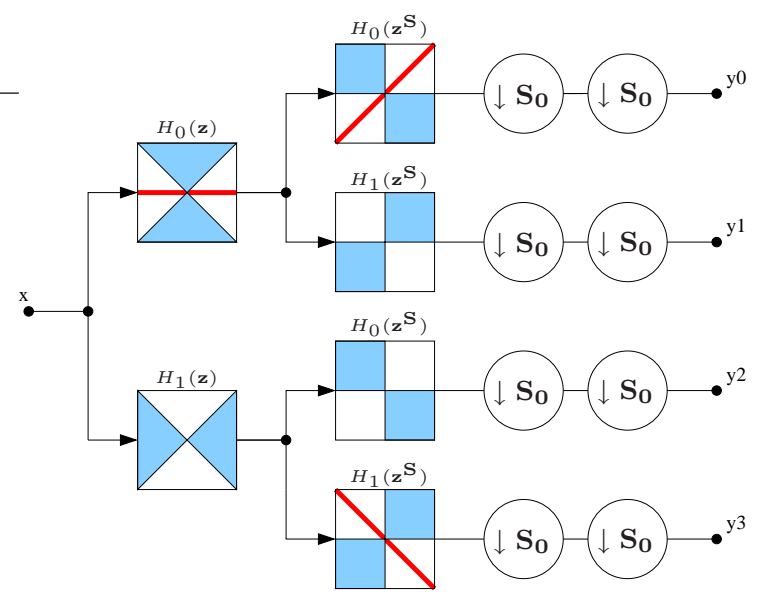

(b) Equivalent DFB

Fig. 8. The DVM directional filter bank. (a) The four-channel DFB with type 0 (horizontal) and type 1 (vertical) DVM filter banks. (b) The four-channel equivalent filter bank. The equivalent filter bank has DVM's in three different directions.

particular node. Furthermore, from Proposition 4, we have that each stage $0 \leq q \leq l$ in the DFB tree introduces DVM's in $2^{q-1}$ channels. Heuristically, we observe that for representing natural images with directional information spread among several orientations, it is desirable to have the set of distinct DVM's as large as possible. Thus, in each DFB stage, the goal is to introduce as many "new" DVM's as possible. For instance, Figure 9 displays three possible arrangements for a $2^{4}$-channel expansion, each with a different number of distinct directions. It can be verified that the arrangement in Figure 9 (a) (Tree 1) yields the maximum number of distinct directions.

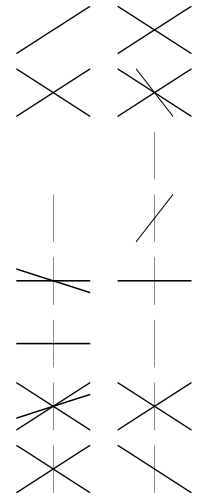

(a) Tree 1

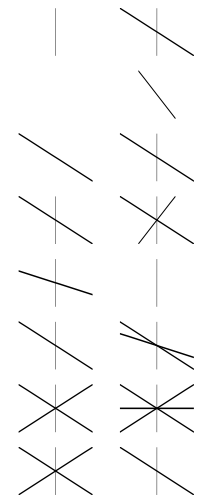

(b) Tree 2

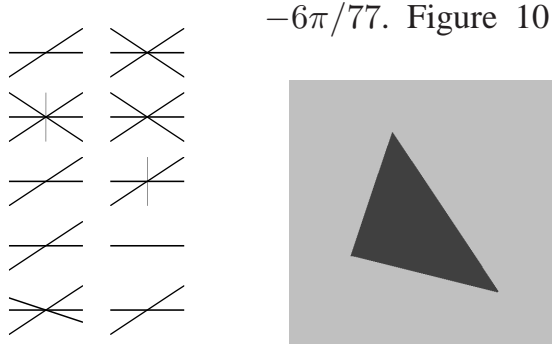

(a) Original

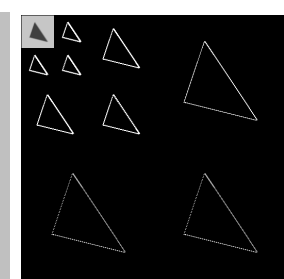

(b) Haar

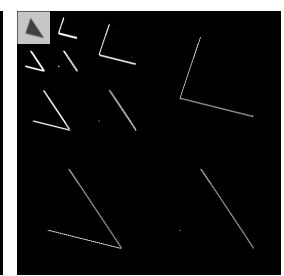

(c) DVM
Fig. 10. Decomposition of synthetic image using two schemes. (a) Original image. (b) Wavelet decomposition. (c) DVM decomposition.

shown is the 4-level decomposition using Haar filters. As the pictures show, the DVM filters produce a more efficient decomposition in the sense that fewer significant coefficients are present. Furthermore, we observe a reduction of about $50 \%$ in the first order entropy after uniformly quantizing the coefficients in the two expansions. Note that the DVM's in the expansion closely matches the edges in the image. For general images, we need DVM's along several directions.

\section{A. Annihilating Directional Edges}

In order to illustrate the potential of the filter banks with DVM's we construct a toy example using Haar-type filters and bi-level images. That is, we use filters with one DVM and 2 nonzero coefficients. As a test image, we use a bilevel polygon image displayed in Figure 10 (a). An efficient

\section{B. Nonlinear Approximation with the Contourlet Transform}

To illustrate the applicability of the directional vanishing moment filters proposed, we perform an experiment in which we replace the conventional DFB in the contourlet transform 


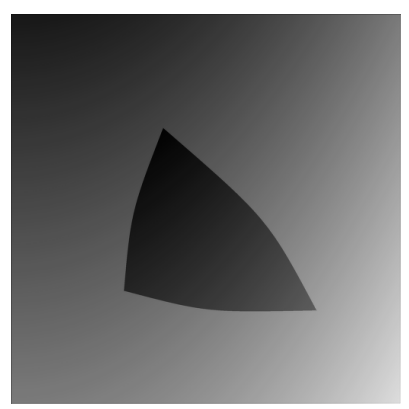

(a)

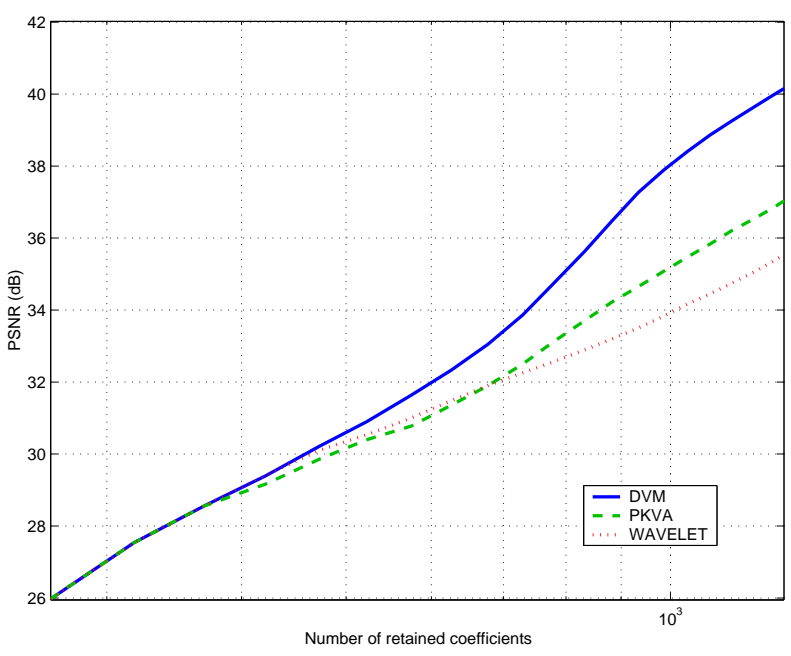

(b) Synthetic

Fig. 11. Nonlinear approximation behavior of the contourlet transform with DVM filters for a toy image. (a) Synthetic piecewise polynomial image. (b) NLA curves (on a semilog scale). This simple toy image is better represented by the contourlet transform with DVM filters.

with a DFB built with DVM fan filter banks following the discussion in Section V (see Figure 8). The filters we use are those designed in Example 1. To study the non-linear approximation (NLA) behavior of the filters in our proposed design, we reconstruct the image using the $N$ coefficients with largest magnitude and compute the resulting PSNR. It is recognized that the faster the asymptotic decay of the NLA error, the sparser the decomposition is. This sparsity is important for potential applications including denoising and compression [6]. The directional expansion tree we use in each scale is one that leads to a maximum number of distinct DVMs and is the same for all test images, hence the expansion is fixed. The analysis filter is zero-phase with $7 \times 13$ coefficients and the synthesis with $9 \times 17$, also zero-phase (see Example 1). As a comparison, we use the quincunx/fan filters of [24] (PKVA), where the analysis filter has $23 \times 23$ taps and the synthesis $46 \times 46$. We observe that the PKVA filters give the best PSNR performance in the contourlet transform among existing designs in the literature.

Figure 11 displays the NLA curve obtained for a piecewise polynomial image. For this synthetic image, a significant improvement is observed. This improvement is due to the fact that the synthetic image has directional information in a very small set of directions, which, due to DVM's, are well represented in the expansion.

Figure 12 shows the NLA curves for the standard $512 \times 512$ "Peppers", and "Barbara" images. As the plots show, the DVM filters slightly improve over PKVA for both natural images. For a highly textured image such as "Barbara" there is significant improvement over wavelets. By contrast, for a smooth image such as "Peppers", the redundancy inherent to the contourlet expansion is more apparent. However, when the number of coefficients is very low, the results are comparable.

Because the DVM filters are considerably shorter, we observe less ringing artifacts when compared against the PKVA filters, even when both give similar PSNR. Figure 13 shows the "Peppers" image reconstructed with 2048 coefficients using both of the filters. As can be seen, the image reconstructed with the DVM filters exhibit less ringing artifacts.

\section{CONCLUSION}

We have studied two-channel biorthogonal filter banks in which one filter bank channel annihilates information along a prescribed direction by means of directional vanishing moments. We investigated in detail the classes of signals that are annihilated by filters having DVM's. In addition, we studied the DVM filter bank design problem and provided a complete characterization of the product filter. The characterization splits the complementary filter into two terms, one minimum order 1-D degenerate filter and a higher order 2-D term. Using the mapping design methodology, we proposed a design procedure in which the mapping can be calculated explicitly. Our approach is easy to carry out and yields a large class of linear-phase 2-D filter banks with DVM's of any prescribed order. We also investigated the potential usage of such filter banks in the context of the contourlet transform. Nonlinear approximation curves indicate that filters with DVM's can be as good as filters designed with frequency response as a primary criteria and, in some cases, yield better results. In addition, because the filters are short, the Gibbs phenomena is considerably reduced.

\section{APPENDIX}

We now show that the Nyquist filters proposed by Phoong et al. in [24] can be seen as a special case of the mapping approach proposed by Tay and Kingsbury [23], and here extended to handle directional vanishing moments. We present the derivation for the quincunx lattice - the rectangular lattice case can be similarly handled. First recall the general form of the 2-D filters obtained in [24]:

$$
\begin{aligned}
& H_{0}\left(z_{1}, z_{2}\right)=\frac{1}{2}\left(z_{1}^{-2 N}+z_{1}^{-1} p\left(z_{1} z_{2}^{-1}\right) p\left(z_{1} z_{2}\right)\right) \\
& H_{1}\left(z_{1}, z_{2}\right)=-p\left(z_{1} z_{2}^{-1}\right) p\left(z_{1} z_{2}\right) H_{0}\left(z_{1}, z_{2}\right)+z_{1}^{-4 N+(2,8)}
\end{aligned}
$$

where $p(z)$ is usually chosen as a halfband filter. The synthesis lowpass filter is $G_{0}\left(z_{1}, z_{2}\right)=-H_{1}\left(-z_{1},-z_{2}\right)$ and, from the above, it follows that

$G_{0}\left(z_{1}, z_{2}\right) H_{0}\left(z_{1}, z_{2}\right)-G_{0}\left(-z_{1},-z_{2}\right) H_{0}\left(-z_{1},-z_{2}\right)=z_{1}^{-6 N+1}$.

Now, consider the delayed versions of $H_{0}(\mathbf{z})$ and $G_{0}(\mathbf{z})$ given by $\widetilde{H}_{0}\left(z_{1}, z_{2}\right)=z_{1}^{2 N} H_{0}\left(z_{1}, z_{2}\right)$ and $\widetilde{G}_{0}\left(z_{1}, z_{2}\right)=$ 


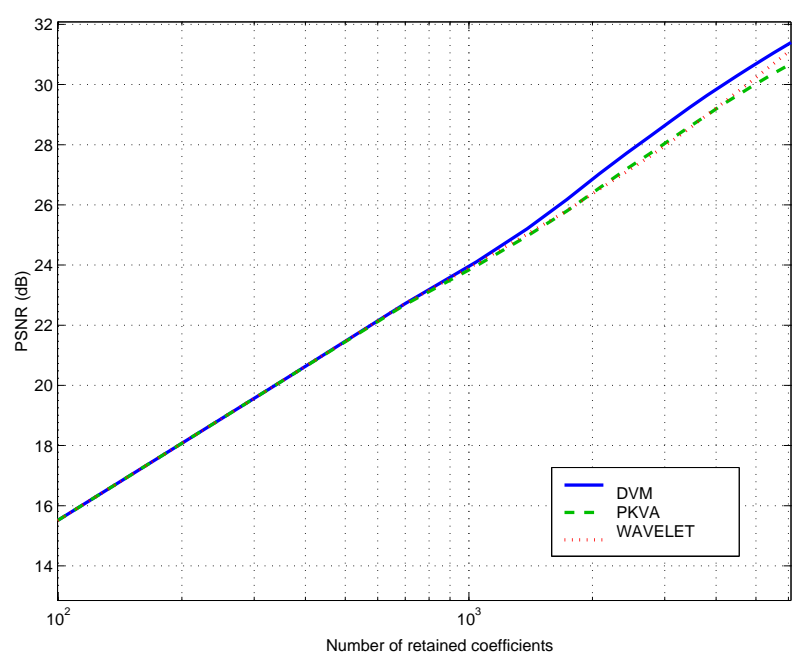

(a) Peppers

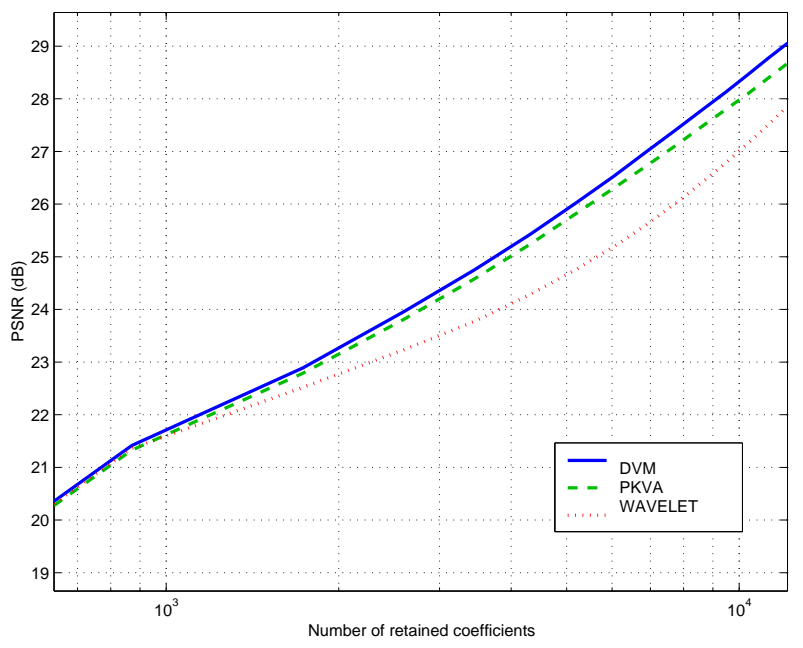

(b) Barbara

Fig. 12. Nonlinear approximation behavior of the contourlet transform with DVM filters for natural images. NLA curves (on a semilog scale) for "Peppers" (a), and "Barbara" (b) images.

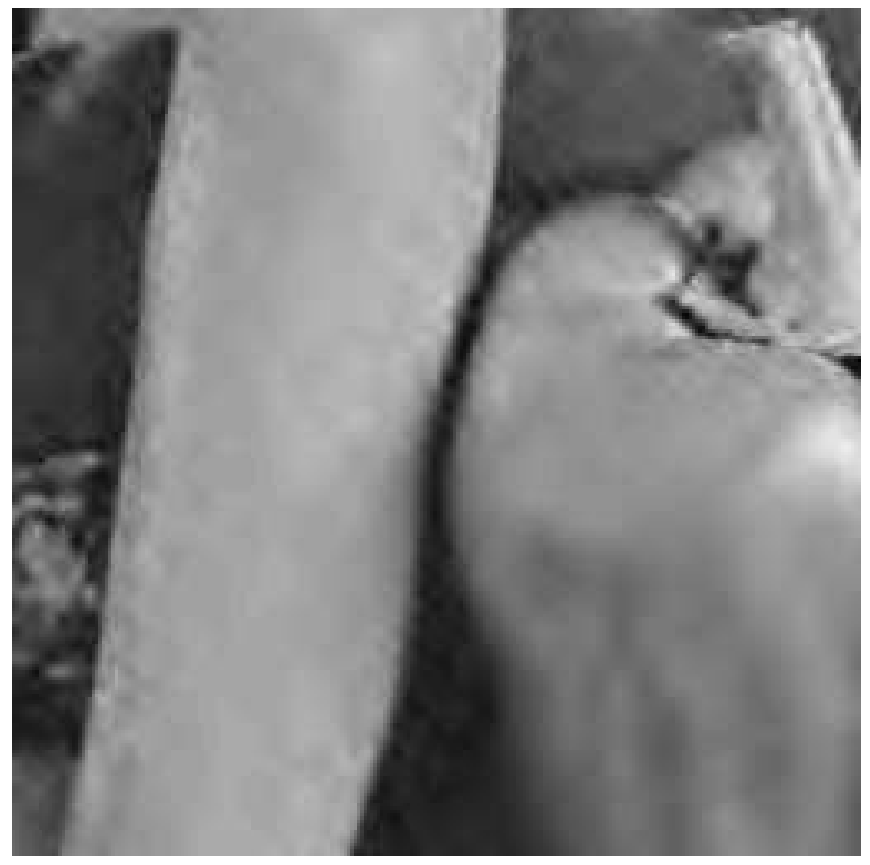

(a) PKVA

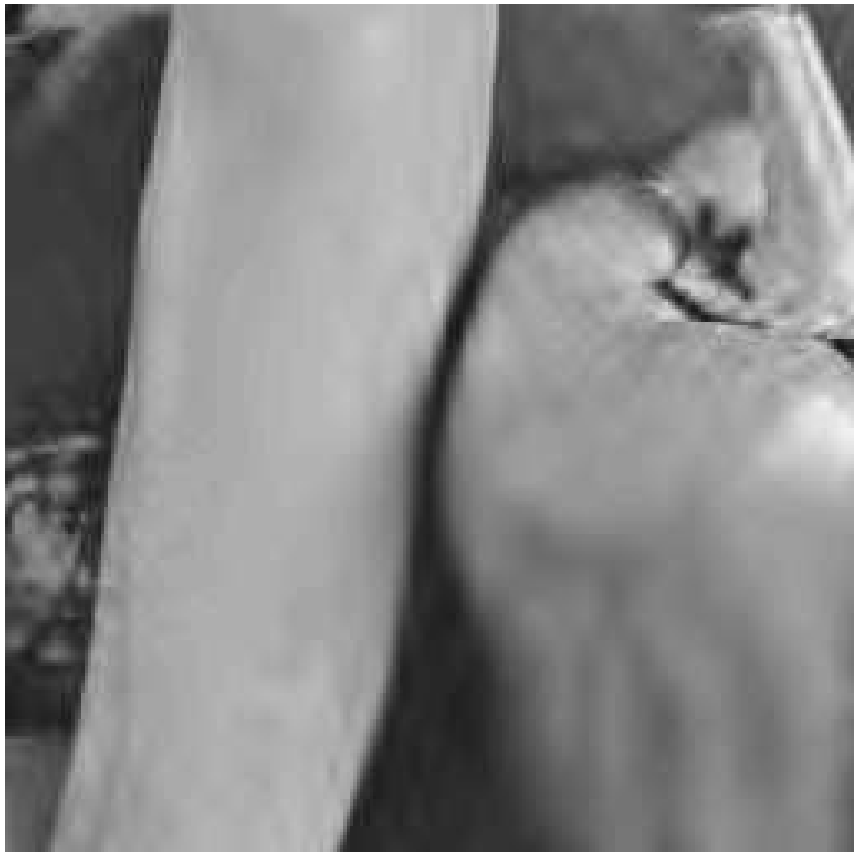

(b) DVM

Fig. 13. "Peppers" image reconstructed with 2048 coefficients. (a) PKVA filters, PSNR $=26.05 \mathrm{~dB}$ (b) DVM filters of Example 1, PSNR $=26.76 \mathrm{~dB}$. The image on the right shows less ringing artifacts.

$z_{1}^{4 N-1} G_{0}\left(z_{1}, z_{2}\right)$. Then

$$
\begin{aligned}
\widetilde{H}_{0}\left(z_{1}, z_{2}\right) & =\frac{1}{2}\left(1+z_{1}^{2 N-1} p\left(z_{1} z_{2}^{-1}\right) p\left(z_{1} z_{2}\right)\right) \\
\widetilde{G}_{0}\left(z_{1}, z_{2}\right) & =\frac{1}{2}\left[2+z_{1}^{2 N-1} p\left(z_{1} z_{2}^{-1}\right) p\left(z_{1} z_{2}\right)\right. \\
& \left.\times\left(1-z_{1}^{2 N-1} p\left(z_{1} z_{2}^{-1}\right) p\left(z_{1} z_{2}\right)\right)\right]
\end{aligned}
$$

The above filters are simply delayed versions of the starting ones, thus being the same filters for practical purposes. Notice that the filters now satisfy $\tilde{H}_{0}(\mathbf{z}) \tilde{G}_{0}(\mathbf{z})+\tilde{H}_{0}(\mathbf{z}) \tilde{G}_{0}(\mathbf{z})=2$. Setting $z:=z_{1}^{2 N-1} p\left(z_{1} z_{2}^{-1}\right) p\left(z_{1} z_{2}\right)$ in (29), we see that the filters can be written in terms of the 1-D polynomials

$$
\begin{aligned}
& H_{0}^{(1 \mathrm{D})}(z)=\frac{1}{2}(1+z) \\
& G_{0}^{(1 \mathrm{D})}(z)=\frac{1}{2}[2+z(1-z)]
\end{aligned}
$$

where $H_{0}^{(1 \mathrm{D})}(z) G_{0}^{(1 \mathrm{D})}(z)+H_{0}^{(1 \mathrm{D})}(-z) G_{0}^{(1 \mathrm{D})}(-z)=2$. Finally note that $z_{1}^{2 N-1} p\left(z_{1} z_{2}^{-1}\right) p\left(z_{1} z_{2}\right)$ is odd regardless of the nature of $p(z)$. Hence we have the following result.

Proposition 7: The filters proposed in [24] constitute a particular case of the mapping design of [23], where the mapping function is $M\left(z_{1}, z_{2}\right)=z_{1}^{2 N-1} p\left(z_{1} z_{2}^{-1}\right) p\left(z_{1} z_{2}\right)$. 


\section{REFERENCES}

[1] E. J. Candès and D. L. Donoho, "New tight frames of curvelets and optimal representations of objects with piecewise $C^{2}$ singularities," Comm. Pure and Appl. Math, vol. 57, no. 2, pp. 219-266, February 2004.

[2] M. N. Do and M. Vetterli, "The contourlet transform: An efficient directional multiresolution image representation," IEEE Trans. Img. Processing, to appear, 2005, http://www.ifp.uiuc.edu/ minhdo/publications.

[3] P. J. Burt and E. H. Adelson, "The Laplacian pyramid as a compact image code," IEEE Trans. Communications, vol. 31, no. 4, pp. 532540, April 1983.

[4] M. N. Do and M. Vetterli, "Framing pyramids," IEEE Trans. Sig. Proc., vol. 51, no. 9, pp. 2329 - 2342, Sept. 2003.

[5] R. H. Bamberger and M. J. T. Smith, "A filter bank for the directional decomposition of images: Theory and design," IEEE Trans. Signal Processing, vol. 40, no. 4, pp. 882-893, April 1992.

[6] S. Mallat, A Wavelet Tour of Signal Processing, 2nd ed. Academic Press, 1999.

[7] M. N. Do, "Directional multiresolution image representations," Ph.D. dissertation, Swiss Federal Institute of Technology, 2001.

[8] Y. Lu and M. Do, "CRISP-contourlet: a critically sampled directional multiresolution representation," in Proc. SPIE Conf. on Wavelets X, San Diego, CA, Aug. 2003.

[9] V. Velisavljević, B. Beferull-Lozano, and M. Vetterli, "Directionlets: regular multi-directional wavelet bases with one-dimensional filtering," ICIP, 2004.

[10] A. L. Cunha and M. N. Do, "Bi-orthogonal filter banks with directional vanishing moments," ICASSP, Philadelphia, PA, 2005.

[11] M. Vetterli, "Wavelets, approximation, and compression," IEEE Signal Proc. Mag., vol. 18, pp. 59-73, Sept. 2001.

[12] G. Strang and T. Nguyen, Wavelets and Filter Banks. MA: WellesleyCambridge Press, 1996.

[13] A. Cohen and I. Daubechies, "Non-separable bidimensional wavelet bases," Rev. Math. Iberoamer, no. 9, pp. 51-137, 1992.

[14] J. Kovačević and M. Vetterli, "Nonseparable multidimensional perfect reconstruction filter banks and wavelet bases for $\mathbb{R}^{n}$," IEEE Trans. Information Theory, vol. 38, no. 2, pp. 533-555, March 1992.

[15] P. Vaidyanathan, Multirate Systems and Filterbanks. Prentice Hall, 1993.

[16] E. Viscito and J. P. Allebach, "The analysis and design of multidimensional FIR perfect reconstruction filter banks for arbitrary sampling lattices," IEEE Trans. Circuits and Systems, vol. 38, no. 1, pp. 29-41, Jan. 1991.

[17] M. Vetterli, "Filter banks allowing perfect reconstruction," Signal Processing, vol. 10, no. 3, pp. 219-244, 1986.

[18] M. Vetterli and C. Herley, "Wavelets and filter banks: Theory and design," IEEE Transactions on Signal Processing, vol. 40, pp. 22072232, Sept. 1992.

[19] I. Daubechies, Ten Lectures on Wavelets. Philadelphia, PA: SIAM, 1992.

[20] R. Ansari, C. W. Kim, and M. Dedovic, "Structure and design of twochannel filter banks derived from a triplet of halfband filters," IEEE Trans CAS-II, vol. 46, no. 12, pp. 1487-1496, December 1999.

[21] J. Kovačević and M. Vetterli, "Nonseparable two- and three-dimensional wavelets," IEEE Trans. Signal Proc., vol. 43, no. 5, pp. 1269-1273, May 1995.

[22] D. Wei and S. Guo, "A new approach to the design of multidimensional nonseparable two-channel orthonormal filter banks and wavelets," IEEE Signal Processing Letters, vol. 7, no. 11, pp. 327-330, November 2000.

[23] D. B. H. Tay and N. G. Kingsbury, "Flexible design of multidimensional perfect reconstruction FIR 2-band filters using transformation of variables," IEEE Trans Img Proc., vol. 2, no. 4, pp. 466-480, October 1993.

[24] S.-M. Phoong, C. W. Kim, P. P. Vaidyanathan, and R. Ansari, "A new class of two-channel biorthogonal filter banks and wavelet bases," IEEE Transactions on Signal Processing, vol. 43, no. 3, pp. 649-661, March 1995.

[25] D. Stanhill and Y. Y. Zeevi, "Two-dimensional orthogonal wavelets with vanishing moments," IEEE Trans. Signal Processing, vol. 44, no. 10, pp. 2579-2590, October 1996.

[26] L. Demanet, "Second generation curvelets," Master's thesis, Université Catholique de Louvain, 2002. 